\title{
Determination of Thymidine Kinase 1 (TK1) Level as a Risk Warning Biomarker to Improve Early Detection of Breast Cancer
}

\author{
Marwa Sameh Abou El-Eneen ${ }^{1, ~ *, ~ H o d a ~ A b o u ~ S e i f ~}{ }^{2}$, Rawia Khalil Fawzy ${ }^{3}$, \\ Abdallah Mohamed Mossa ${ }^{4}$ \\ ${ }^{1}$ Radiation Sciences Department, Medical Research Institute, Alexandria University, Alexandria, Egypt \\ ${ }^{2}$ Pathology Department, Medical Research Institute, Alexandria University, Alexandria, Egypt \\ ${ }^{3}$ Radiodiagnosis Department, Medical Research Institute, Alexandria University, Alexandria, Egypt \\ ${ }^{4}$ Techno Scan Radiology Centre, Damietta, Egypt
}

Email address:

marwa.aboueleneen@alexu.edu.eg(M. S. A. El-Eneen)

${ }^{*}$ Corresponding author

\section{To cite this article:}

Marwa Sameh Abou El-Eneen, Hoda Abou Seif, Rawia Khalil Fawzy, Abdallah Mohamed Mossa. Determination of Thymidine Kinase 1 (TK1) Level as a Risk Warning Biomarker to Improve Early Detection of Breast Cancer. Cancer Research Journal. Vol. 7, No. 4, 2019 , pp. $161-166$. doi: $10.11648 /$ j.crj.20190704.17

Received: November 22, 2019; Accepted: December 12, 2019; Published: December 24, 2019

\begin{abstract}
Early detection of breast cancer (BC) is a global target to reduce mortality and morbidity also to improve therapeutic and survival outcomes. Currently, mammography is the gold standard in BC diagnosis followed by biopsy when warranted. Thymidine Kinase 1 (TK1) is a proliferative biomarker that succeeded in discovering premalignant transformations of breast cancer before the appearance of any symptoms. This study aimed to provide a non-invasive method to early detect $\mathrm{BC}$ by measuring TK1 in sera of women with breast lesions alongside mammography. The study included 271 women divided into five BIRADS categories. Methods and Material: only one blood sample was collected from each woman to detect TK1 concentration, before undergoing mammography and Fine Needle Aspiration Cytology (FNAC) or true cut. Results: TK1 levels were significantly different between BI-RADS categories. It was correlated with clinical stage, histological grade, lymph node metastasis, and vascular invasion. TK1 levels could distinguish between healthy individuals and patients who had breast lesions with a sensitivity and a specificity as follows 91.3 and $87.5 \%$, respectively. Furthermore, this test could discriminate between benign and malignant breast lesions with a sensitivity of $92.5 \%$ and a specificity of $91.2 \%$. Conclusion: These findings suggest the determination of TK1 levels as a risk warning biomarker to improve early detection of BC.
\end{abstract}

Keywords: BI-RADS, Thymidine Kinase 1 (TK1), Breast Cancer, Benign, Proliferative Marker

\section{Introduction}

Breast cancer $(\mathrm{BC})$ is considered the second major cancer among women worldwide [1]. Late diagnosis of $\mathrm{BC}$ reduces survival and quality of life [2]. Thus, early detection of the tumor is a prerequisite to effective treatment and to minimize morbidity and mortality rates of $\mathrm{BC}$, as an early diagnosis in a localized site increases 5-year survival rates to $98 \%[3,4]$.

Currently, mammography is the gold standard in breast cancer diagnosis followed by biopsy when warranted [2]. The Breast Imaging and Reporting Data System (BI-RADS $®)$ is a number system that categorizes mammography results into seven categorizes from 0 through 6 [5]. The most difficult assessment category for the radiologists is BI-RADS 3 (probably benign) [6]. As many previous studies displayed significant differences between radiologists' interpretation of breast lesions as BI-RADS 3 [7].

Patients are assigned as a BI-RADS 3 should undergo follow up imaging for 2 years concerning a 6-month interval to document steadiness or resolve of a finding [8]. However, if 6-month intervals imaging follows up of BI-RADS 3 results in undetermined radiological lesions, this can initiate anxiety for radiologists, women, and breast surgeons. Also, this follow up 
is ineffective when patients do not return for follow-up imaging and that leads to delayed in diagnosis if the progression of lesions occurred over time. Additionally, repeated exposure to ionizing radiation increases the risk of cancer and is costly [8-11].

Therefore, there is a necessity for non-invasive biomarkers that identify precancerous breast lesions. Thymidine kinase 1 is a proliferative biomarker that is associated with a cellular division and intended to be a tumor growth marker [3, 12, 13]. It controls the intracellular thymidine pool via salvage pathway, as it catalyzes the transfer of phosphate group from ATP to $5^{\prime}$ hydroxyl group of thymidine to create thymidine monophosphate which is then incorporated in DNA replication [13]. TK1 levels depend on cell cycle considering that its levels are hardly detected in resting cells, but once passing G1 checkpoint its levels increased, reaching the maximum at $\mathrm{S}$ phase then disappeared at mitosis [13].

Therefore, this study aimed to provide a non-invasive method to early detect BC by measuring TK1 in sera of women with breast lesions alongside mammography.

\section{Subjects and Methods}

\subsection{Study Design and Subjects}

This study included 271 females divided into five categories according to BI-RADS classification. Only one blood sample was collected from each woman enrolled in the study to detect TK1 concentration, before undergoing mammography and Fine Needle Aspiration Cytology (FNAC) or true cut, from January 2014 to June 2017.

All subjects were categorized as either BI-RADS 1, 2, 3, 4, or 5 at the time of recruitment, as determined by mammography, ultrasound, or combination of both modalities if needed. All BI-RADS 3 subjects were followed for three years with 6 months interval by using imaging techniques and pathological examination if necessary. Each woman who participated in this study signed an informed consent form preceding inclusion in the study protocol. This study was approved by the local Ethical Committee. Control subjects had one mammogram minimally with normal results and no history of cancer, while cancer patients did not have previous cancer nor received treatment before sample collection.

We excluded subjects if they had a bacterial or viral infection at presentation, their follow-up was not possible, or they were reluctant to present written informed consent, or their samples were haemolysed.

Tumor stage was categorized according to the TNM-classification, which was conducted correspondingly to the WHO System [14]. The histopathological grading was classified according to the Bloom and Richardson system classification [15]. The characteristics of the subjects were represented in Tables $(1 \& 2)$.

\subsection{The Detection of TK1}

Blood samples were collected using serum separator tubes and clotted for one hour at room temperature then centrifuged for 15 minutes at $1000 \mathrm{~g}$ then the supernatant sera were fractionated and stored at $-80^{\circ} \mathrm{C}$.

The concentrations of serum TK1 were measured by using a Thymidine Kinase 1 Enzyme-linked Immunosorbent Assay (ELISA) (commercial kit; MyBiosource, Inc., USA). The procedure was implemented according to the manufacturer's protocol; $100 \mu 1$ standard or sample was added to each well then incubated 2 hours at $37^{\circ} \mathrm{C}$. The plate was aspirated then $100 \mu$ of Biotin-antibody $(1 \mathrm{x})$ was put in each well then incubated 1 hour at $37^{\circ} \mathrm{C}$.

Each well was aspirated and washed then $100 \mu 1$ of HRP-avidin (1x) was added and incubated 1 hour at $37^{\circ} \mathrm{C}$. Each well was aspirated and washed then $90 \mu \mathrm{l}$ of TMB Substrate was added to each well and incubated for 15-30 minutes at $37^{\circ} \mathrm{C}$. $50 \mu \mathrm{l}$ of Stop Solution was put then read at $450 \mathrm{~nm}$ using $800^{\mathrm{TM}}$ TS Absorbance Reader (Bio Tek Instruments, Inc., USA). Results were expressed as $\mathrm{pg} / \mathrm{ml}$. The analytic sensitivity was $15.6 \mathrm{pg} / \mathrm{ml}$. The coefficients of variation within- and between-assays were $<8 \%$ and $<10 \%$, respectively.

Table 1. Subject characteristics.

\begin{tabular}{ll}
\hline Characteristics & No. $(\%)$ \\
\hline Age (Years) & \\
Min. - Max. & $24.0-87.0$ \\
Mean \pm SD. & $41.4 \pm 10.5$ \\
Median & 41.0 \\
Menopausal Status & \\
Premenopausal & $189(69.7)$ \\
Perimenopausal & $52(19.2)$ \\
Postmenopausal & $30(11.1)$ \\
BI-RADS & \\
1 & $40(14.8)$ \\
2 & $41(15.1)$ \\
3 & $100(36.9)$ \\
4 & $40(14.8)$ \\
5 & $50(18.4)$ \\
\hline
\end{tabular}

Table 2. Clinicopathological characteristics of breast cancer patients.

\begin{tabular}{ll}
\hline Characteristics & No. (\%) \\
\hline Clinical Stage & \\
I & $19(17.8)$ \\
II & $43(40.2)$ \\
III & $45(42.0)$ \\
Histological Grade & \\
I & $18(16.8)$ \\
II & $47(43.9)$ \\
III & $42(39.3)$ \\
Lymph Nodes & \\
+ Ve & $79(73.8)$ \\
-Ve & $28(26.2)$ \\
Vascular invasion & \\
+ Ve & $76(71.0)$ \\
-Ve & $31(29.0)$ \\
\hline
\end{tabular}

\subsection{Statistical Analysis}

IBM SPSS software package version 20.0 was used to analyze data. The Kolmogorov-Smirnov test was utilized to verify the normality of distribution. Quantitative data were described using interquartile range (IQR), median, mean and standard deviation. The significance of the obtained results 
was judged at the 5\% level. Chi-square test, F-test (ANOVA), Receiver operating characteristic curve (ROC) and Spearman coefficient were used [16, 17].

\section{Results}

\subsection{The Study Participants}

An overall of 271 women was volunteered for this study with a mean age of $41.4 \pm 10.5$ years. The majority of them were in premenopausal status $(69.7 \%)$ and most breast cancer patients had lymph nodes and vascular invasion $(74.8 \%$ and $71 \%$, respectively). After three years follow up, there were twenty-eight patients with BI-RADS category 3 developed cancer (28/100), after performing fine-needle aspiration cytology. The total number of BC patients was 107 patients.
The clinicopathological characteristics of all women enrolled in the study, including age, menopausal status, BI-RADS categories, and $\mathrm{BC}$ patients involving histologic grade, lymph node metastasis, TNM stage, and vascular invasion are summarized in Tables ( $1 \& 2)$.

\subsection{The Concentration of TK1 in Different BI-RADS Categories}

There was a highly statistically significant difference in the concentration of TK1 between all BI-RADS categories $(1,2,3$, 4 and 5) as mean \pm SD were $158.7 \pm 15.52,207.6 \pm 23.60$, $227.8 \pm 44.55, \quad 251.3 \pm 22.46$ and $311.5 \pm 43.72 \mathrm{pg} / \mathrm{ml}$, respectively, $\mathrm{P}<0.001$. BI-RADS category 1 represented the control group as it was negative for benign and malignant lesions, Figure 1.

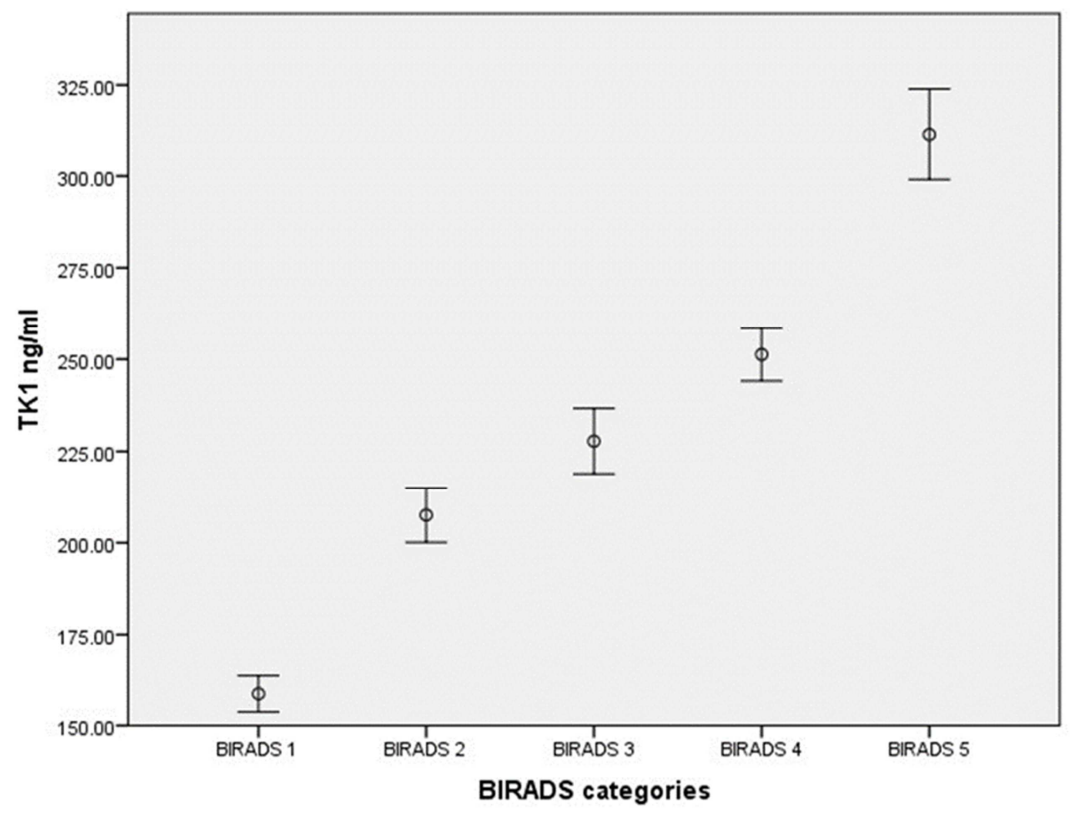

Figure 1. Comparison between different BI-RADS categories dependent on TK1 concentration (mean $\pm S D)$.

\subsection{The Correlation of TK1 in Patients with Breast Lesions}

There was a positive significant correlation between TK1 concentrations and the clinical stage, histological grade, lymph node metastasis and vascular invasion $\left(\mathrm{r}_{\mathrm{s}}=0.321,0.315\right.$, 0.344 and 0.278 and $\mathrm{P}=0.001, \mathrm{P}=0.001, \mathrm{P}<0.001$, and $\mathrm{P}=0.004$, respectively). While there was no correlation of TK1 levels versus age and menopausal status $\left(r_{\mathrm{s}}=0.110\right.$ and 0.087 also, $\mathrm{P}=0.070$ and 0.152 , respectively).

\subsection{Receiver Operating Characteristics (ROC) Curve}

Area under the curve (AUC) for ROC curve that differentiated between BI-RADS category 1 and the BI-RADS categories (2, 3, 4 and 5) was 0.973 at a cut off value 175 $\mathrm{pg} / \mathrm{ml}$, the sensitivity and the specificity was $91.3 \%$ and $87.5 \%$, respectively, Table 3, Figure $2 \mathrm{~A}$. While the AUC for the ROC curve that differentiated between benign and malignant breast lesions was 0.968 at a cut off value of $240 \mathrm{pg} / \mathrm{ml}$, the sensitivity and the specificity was $92.5 \%$ and $91.2 \%$, respectively, Table 3, Figure $2 \mathrm{~B}$.

TK1 concentrations of the 28 cases who developed cancer during follow up in BI-RADS category 3 were higher than the cut off value $240 \mathrm{pg} / \mathrm{ml}$.

Table 3. Results from the ROC curve analysis between different BI-RADS categories dependent on TK1 concentration.

\begin{tabular}{|c|c|c|c|c|c|c|c|c|c|}
\hline Groups & Youden Index & Cut off (pg/ml) & $\operatorname{AUC}(\mathrm{CI})$ & $\mathbf{P}$ & Sn & Sp & PPV & NPV & $\mathrm{ACC}$ \\
\hline $\begin{array}{l}\text { BI-RADS } 1 \text { vs, } \\
\text { BI-RADS 2, 3, } 4 \text { and } 5\end{array}$ & 0.788 & 175 & $0.973 *(0.955-0.991)$ & $<0.001^{*}$ & 91.3 & 87.5 & 98.17 & 67.92 & 92.25 \\
\hline Benign vs, Malignant & 0.837 & 240 & $0.968 *(0.949-0.987)$ & $<0.001 *$ & 92.5 & 91.2 & 89.19 & 95.00 & 92.62 \\
\hline
\end{tabular}

$\mathrm{P}=$ probability, $\mathrm{Sn}=$ Sensitivity, $\mathrm{Sp}=$ Specificity, $\mathrm{ACC}=$ Accuracy. 


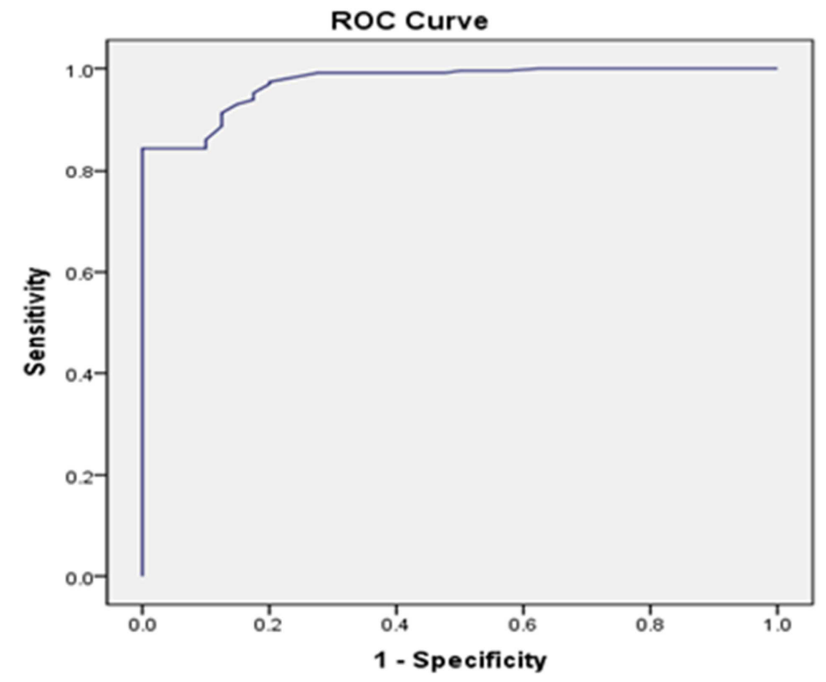

A

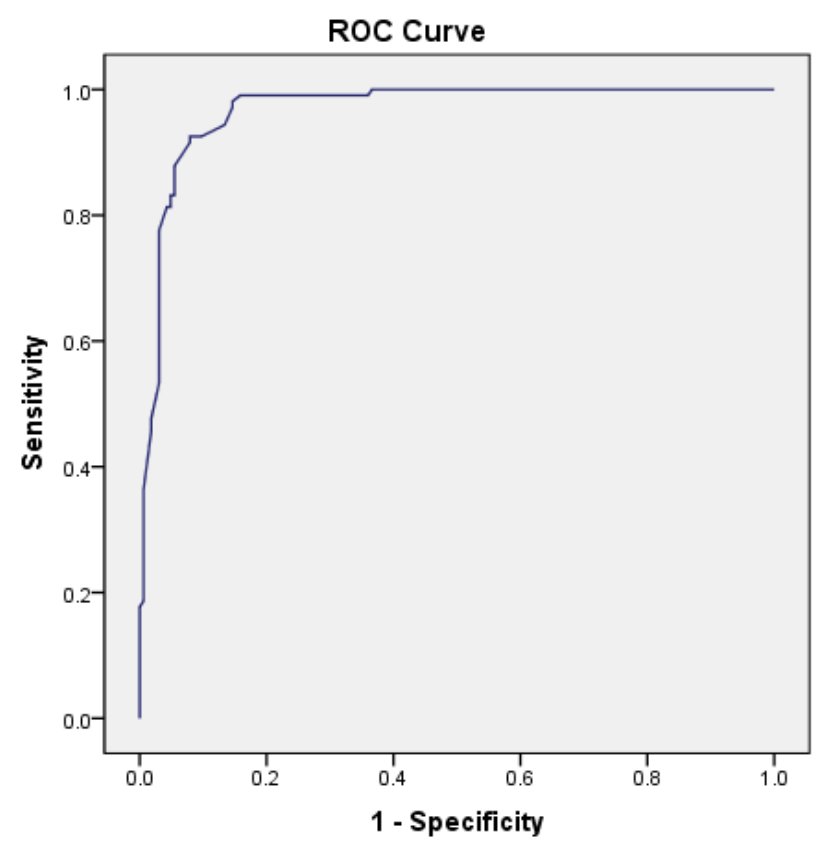

B

Figure 2. ROC curve analysis for TK1. A. to differentiate between BI-RADS 1 and BI-RADS 2, 3, 4 and 5. B. to differentiate between benign and malignant breast lesions.

\section{Discussion}

Early detection of $\mathrm{BC}$ is a global target for Breast Health Global Initiative (BHGI) and World Health Organization (WHO) particularly in low and middle- income countries to reduce mortality and morbidity also to improve therapeutic and survival outcomes $[18,19]$.

The non-invasive serological biomarkers may provide data about breast lesions of probable malignancy before the assessment by imaging techniques [20].

Thymidine Kinase 1 is one of the proliferative biomarkers used previously in many screening tests for early detection of cancer. It succeeded in discovering premalignant transformations of breast cancer before the appearance of any symptoms [20].

In the present study, we observed that the highest levels of TK1 were found in breast cancer patients and the lowest levels were found in the normal individuals. Also, it could discriminate between benign and malignant patients. This was accepted by many previous studies [13, 20, 21].

Our work is the first one to combine between mammography techniques, as a gold standard in the early detection of breast cancer and determination of the concentration of TK1 levels. This simple TK1 test could differentiate between all BI-RADS categories from 1 through 5 including BI-RADS 3 which causes perplexity for radiologists to be assessed [6]. As during follow up of BI-RADS 3, twenty-eight patients who had high TK1 levels developed malignancy later; $82.1 \%$ were invasive ductal carcinoma, $14.3 \%$ were ductal carcinoma in situ and $3.6 \%$ were hepatocellular carcinoma.

Consequently, TK1 could be used in the detection of BC in the premalignant state. Huang S. et. al. [20] and Chen ZH et. al [11]. noticed high TK1 levels in premalignant conditions and the early stages of BC. Previously, TK1 used in screening health programs of cancer and recommended the use of TK1 as a risk predictor biomarker for malignant transformation [11, 21, 22].

Cao X. et. al. [21], Huang S. et. al. [20] Dedicated that TK1 levels were not affected by age of patients and that was in line with our results. Nisman B. et. al. [23] Were found that TK1 levels were not correlated with menopausal status and similarly noticed in this study. Subsequently, it could be used to early assess BC whatever the age of women.

Protein levels of TK1 were positively correlated with lymph node metastasis, clinical stage and histological grade of $\mathrm{BC}$ patients which reflected the diagnostic and prognostic value of TK1. A majority of studies to date advocate this correlation where He E. et. al. [24] Confirmed a correlation between tumor and serum TK1 levels with both stage and grade. Kumar J. K. et. al. [25] found elevated TK1 levels in metastatic lymph node patients versus those without these complications.

The mechanism responsible for the discrepancy in TK1 levels among BI-RADS categories and between malignant, benign and normal individuals is still unclear. But there were some possible interpretations; first, due to the presence of a factor in serum modified TK1's stability. Second, normal cells die in the G0 or early G1 in the cell cycle where the levels of TK1 were down, while cancer cells die in the $\mathrm{S} / \mathrm{G} 2$ phase where TK1 levels were up, consequently liberating more TK1 in serum. Also, there was a difference in the clearance of TK1 between normal and malignant cells [11].

In the present study, AUC, sensitivity, and specificity at a cut off $175 \mathrm{pg} / \mathrm{ml}$ were $0.973,91.3 \%$ and $87.5 \%$, respectively, this was in agreement with Chen $\mathrm{ZH}$ et. al. [11] that detected AUC and sensitivity were 0.94 and $94.3 \%$, respectively. This indicated that TK1 had high precision in early detecting $\mathrm{BC}$, since $0.9 \geq \mathrm{AUC} \leq 1$.

Increasing cut off value to $240 \mathrm{pg} / \mathrm{ml}$ to differentiate 
between benign and malignant patients resulted in changing of AUC, sensitivity, and specificity as follows $0.968,92.5 \%$ and $91.2 \%$, respectively.

Since BI-RADS 3 was set as a problematic category in BI-RADS Atlas [5] and needed repeating mammography during follow up till assessing upgrading or downgrading of BI-RADS 3 patients, which may cause anxiety/delay in detection, thus adjunction with non-invasive determination of serum TK1 may improve early assessment of BI-RADS 3 and reduce exposure to $\mathrm{X}$-ray.

\section{Conclusion}

Serum TK1 could detect premalignant transformation before assessment by imaging techniques with a non-invasive, fast, easy, specific and sensitive method. TK1 levels were significantly different among BI-RADS categories. It was strongly positively correlated with clinical stage, histological grade, lymph node metastasis, and vascular invasion. Measuring TK1 in sera of patients that were assigned as BI-RADS 3 may help in upgrading or downgrading this difficult assessment category. These findings suggest the determination of TK1 levels as a risk warning biomarker to improve early detection of $\mathrm{BC}$, enhance accuracy in the assessment of BI-RADS 3 and reduce repeated exposure to $\mathrm{X}$-ray. According to our results, we recommended performing FNAC or true cut for BI-RADS 3 patients with high TK1 levels.

Furthermore, this research needed to be applied to a larger number of BI-RADS 3 cases and follow up women with BI-RADS 1 and 2 who had higher concentrations of TK1 to early detect $\mathrm{BC}$ and to reduce their risk to breast cancer. Also, try to study the molecular mechanisms responsible for the elevation of TK1 levels.

\section{References}

[1] Ghoncheh M, Pournamdar Z, Salehiniya H. Incidence and Mortality and Epidemiology of Breast Cancer in the World. Asian Pac J Cancer Prev. 2016; 17: 43-6.

[2] Pezuk JA, Miller TLA, Bevilacqua JLB, de Barros ACSD, de Andrade FEM, E Macedo LFA, et. al. Measuring plasma levels of three microRNAs can improve the accuracy for identification of malignant breast lesions in women with BI-RADS 4 mammography. Oncotarget. 2017; 8: 83940-83948.

[3] Chen CC, Chang TW, Chen FM, Hou MF, Hung SY, Chong IW, et al. Combination of multiple mRNA markers (PTTG1, Survivin, UbcH10 and TK1) in the diagnosis of Taiwanese patients with breast cancer by membrane array. Oncology 2006; 70: $438-446$.

[4] American Cancer Society. Breast cancer facts and figures 2017-2018. Atlanta, GA: American Cancer Society; 2017.

[5] Mendelson EB, Böhm-Vélez M, Berg WA, Whitman GJ, Feldman MI, Madjar H, D’Orsi CJ, Sickles EA, Morris EA. ACR BI-RADS ${ }^{\circledR}$ Atlas, Breast Imaging Reporting and Data System. Reston (VA): American College of Radiology; 2013.
[6] Lee KA, Talati N, Oudsema R, Steinberger S, Margolies LR. BI-RADS 3: Current and Future Use of Probably Benign. Curr Radiol Rep 2018; 6: 5.

[7] Michaels AY, Chung CSW, Frost EP, Birdwell RL, Giess CS. Interobserver variability in upgraded and non-upgraded BI-RADS 3 lesions. Clin Radiol 2017; 72: e1-e6.

[8] Salamaa D H, Gewefelb H. BI-RADS 3 Breast Lesions: Can Follow-up Replace Biopsy. Egypt J Hosp Med 2014; 54: 110.

[9] Hot S, Coşkun ZÜ, Akçakaya A, Bender Ö, Türkmen ÜA, Nayır PÖ, Sarı A, Hot AB. The breast lesion excision system procedure: An optimal solution for the management of indeterminate BI-RADS category 3 breast lesions in women with severe anxiety. Saudi Med J. 2018; 39: 891-896.

[10] Pauwels EK, Foray N, Bourguignon MH. Breast Cancer Induced by X-Ray Mammography Screening? A Review Based on Recent Understanding of Low-Dose Radiobiology. Med Princ Pract. 2016; 25: 101-9.

[11] Chen ZH, Huang SQ, Wang Y, Yang AZ, Wen J, Xu XH, et al. Serological thymidine kinase 1 is a biomarker for early detection of tumours --a health screening study on 35,365 people, using a sensitive chemiluminescent dot blot assay. Sensors (Basel). 2011; 11: 11064-80.

[12] Jagarlamudi KK, Wang L, Eriksson S. Doxorubicin effects on leukemia and breast cancer cells in culture on the TK1 protein levels using AroCell TK 210 ELISA: a tool for drug development. Nucleosides Nucleotides Nucleic Acids. 2018; 37: 679-686.

[13] Jagarlamudi KK, Hansson LO, Eriksson S. Breast and prostate cancer patients differ significantly in their serum Thymidine kinase 1 (TK1) specific activities compared with those hematological malignancies and blood donors: implications of using serum TK1 as a biomarker. BMC Cancer. 2015; 15: 66.

[14] Percy C, Fritz A, Jack A, Shanmugarathan S, Sobin L, Parkin $\mathrm{DM}$, et al. International Classification of Diseases for Oncology (ICD-O). 3rd ed. Geneva: World Health Organization; 2000.

[15] Elston EW, Ellis IO. Method for grading breast cancer. J Clin Pathol. 1993; 46: 189-90.

[16] Kotz S, Balakrishnan N, Read CB, et al. (2006) Encyclopedia of statistical sciences. 2nd ed. Hoboken, N. J.: Wiley-Interscience.

[17] Kirkpatrick LA, Feeney BC (2013) A simple guide to IBM SPSS statistics for version 20.0. Student ed. Belmont, Calif: Wadsworth, Cengage Learning.

[18] Anderson BO, Shyyan R, Eniu A, Smith RA, Yip CH, Bese NS, et al. Breast cancer in limited-resource countries: an overview of the Breast Health Global Initiative 2005 guidelines. Breast J. 2006; 12 Suppl 1: S3-15.

[19] World Health Organization. Cancer control: knowledge into action: WHO guide for effective programmes: Module 3: Early Detection: World Health Organization; 2007.

[20] Huang S, Lin J, Guo N, Zhang M, Yun X, Liu S, et al. Elevated serum thymidine kinase 1 predicts risk of pre/early cancerous progression. Asian Pac J Cancer Prev. 2011; 12: 497-505. 
[21] Cao X, Wang Y, Yang P, Zhou H, Liu C, Chen Z. Application of serum thymidine kinase 1 of 26055 cases in health screening for early detection of premalignant/early malignant tumors. Zhong Nan Da Xue Xue Bao Yi Xue Ban. 2014; 39: 1029-34.

[22] Chen Z, Zhou H, Li S, He E, Hu J, Zhou J, et al. Serological thymidine kinase 1 (STK1) indicates an elevated risk for the development of malignant tumours. Anticancer Res. 2008; 28 (6B): 3897-907.

[23] Nisman B, Kadouri L, Allweis T, Maly B, Hamburger T, Gronowitz $\mathrm{S}$, et al. Increased proliferative background in healthy women with BRCA1/2 haploinsufficiency is associated with high risk for breast cancer. Cancer Epidemiol Biomarkers Prev. 2013; 22: 2110-5.
[24] He E, Xu XH, Guan H, Chen Y, Chen ZH, Pan ZL, et al. Thymidine kinase 1 is a potential marker for prognosis and monitoring the response to treatment of patients with breast, lung, and esophageal cancer and non-Hodgkin's lymphoma. Nucleosides Nucleotides Nucleic Acids. 2010; 29: 352-8.

[25] Kumar JK, Aronsson AC, Pilko G, Zupan M, Kumer K, Fabjan $\mathrm{T}$, et al. A clinical evaluation of the TK 210 ELISA in sera from breast cancer patients demonstrates high sensitivity and specificity in all stages of disease. Tumour Biol. 2016; 37: 11937-11945. 